Relato de Caso

\title{
Solução Minimamente Invasiva para Mancha Branca Idiopática Através da Microabrasão do Esmalte: Relato de Caso Clínico
}

\author{
Minimally Invasive Solution for Idiopathic White Stain Through Enamel Microbrain: \\ Clinical Case Report
}

Wanêssa Trigueiro Casimiro1; Germana Coeli de Farias Sales²; Rosenês Lima dos Santos²

${ }^{1}$ Graduanda do curso de Odontologia da Universidade Federal da Paraíba, João Pessoa-PB; 2Professora do curso de Odontologia da Universidade Federal da Paraíba, João Pessoa-PB

Autor e endereço para correspondência:

Rosenês Lima dos Santos - Departamento de Clínica e Odontologia Social, Centro de Ciências da Saúde, Universidade Federal da Paraíba, Campus I, João Pessoa, PB - Brasil - CEP: 58051900. Email: roseneslima@hotmail.com

\begin{abstract}
Resumo
Introdução: A técnica de microabrasão do esmalte vem sendo utilizada pelos cirurgiões dentistas, como solução para reestabelecer a estética dental. A microabrasão do esmalte é a remoção de manchas ou irregularidades na superfície do esmalte por utilização de substâncias ácidas associadas à pasta microabrasiva. $\mathrm{O}$ objetivo desse trabalho é relatar um caso clínico realizado através da microabrasão do esmalte no elemento $23 \mathrm{com}$ a presença de mancha branca idiopática. Relato de caso: Paciente A. Z., 22 anos, sexo feminino, compareceu a Clínica de Dentística II da Universidade Federal da Paraíba com queixa do aspecto estético do elemento 23, devido a presença de mancha branca. No exame clínico intra-oral, verificou-se a presença de manchas brancas na face vestibular e com um pequeno envolvimento da face mesial do elemento 23. Para avaliar a profundidade da mancha foi utilizado o método de transiluminação. Com esse método, pode-se perceber que as manchas eram pouco profundas. Sendo assim, o diagnóstico sugestivo desse caso foi de opacidade demarcada idiopática. Optou-se pelo tratamento através da Microabrasão do Esmalte Dental com o uso de Ácido clorídrico a 12\% + Carbeto de silício, por ser um composto microabrasivo indicado para remoção de manchas e defeitos de desenvolvimento na formação do esmalte. Conclusão: A técnica de microabrasão empregada atendeu a necessidade estética da paciente; e demonstrou ser eficaz, conservadora e segura para remoção de manchas superficiais no esmalte dental.
\end{abstract}

Palavras-chave: Esmalte Dentário. Estética. Microabrasão do Esmalte. 


\begin{abstract}
Introduction: The technique of enamel microabrasion has been used by dental surgeons as a solution to reestablish dental aesthetics. The microabrasion of the enamel is the removal of stains or irregularities on the surface of the enamel by the use of acidic substances associated with the microabrasive paste. The objective of this work is to report a clinical case performed through the microabrasion of the enamel in element 23 with the presence of idiopathic white stain. Case report: Patient A. Z., 22 years old, female, showed up at the Dentistry Clinic II of the Federal University of Paraíba complaining about the aesthetic aspect of element 23 , due to the presence of white stain. In the intra-oral clinical examination, there were white stains on the vestibular surface and a small involvement of the mesial face of the element 23 . To evaluate the depth of the stain, the transillumination method was used. With this method, one can see that the stain was not very superficial. Thus, the suggestive diagnosis of this case was idiopathic marked opacity. We opted for the treatment through Microabrasion of Dental Enamel with the use of $12 \%$ Hydrochloric Acid + Silicon carbide, because it is a microabrasive compound indicated for the removal of blemishes and defects of development in the enamel formation. Conclusion: The microabrasion technique applied met the aesthetic need of the patient; and has been shown to be effective, conservative and safe for removal of superficial patches on tooth enamel.
\end{abstract}

Keywords: Dental Enamel. Aesthetics. Enamel Microabrasion.

\title{
Introdução
}

A procura por tratamentos estéticos tem aumentado em todas as áreas da Odontologia. As pessoas têm procurado obter um sorriso harmônico e consequentemente uma boa aparência. Nas últimas décadas, houve um grande avanço tecnológico na área de materiais restauradores estéticos, como também a inovação de novas técnicas para remoção de manchas que possam acometer os substratos dentários ${ }^{1,2}$.

Uma solução que vêm sendo utilizada pelos cirurgiões dentistas a fim de recuperar a estética do paciente é a técnica de microabrasão do esmalte. Esta é uma alternativa estética eficaz para remoção de manchas de esmalte, de fácil aplicação, é conservadora, minimamente invasiva, com pouco desgaste de esmalte e com resultado estético satisfatório quando corretamente indicada ${ }^{3-5}$.

A microabrasão do esmalte é a remoção de manchas ou irregularidades na superfície do esmalte por associação de substâncias ácidas associadas à pasta microabrasiva, por meio de esfregaço e, pode essa técnica ser utilizada sozinha ou em combinação com outros meios como clareamento dental e uso de materiais restauradores ${ }^{6,7}$. Essa técnica está indicada para manchas 
resultantes de distúrbios de desenvolvimento como fluorose, hipoplasia, amelogênese imperfeita, cáries paralisadas e/ou defeitos estruturais que pigmentam o esmalte, pós remoção de bráquetes ortodônticos, dentes naturalmente escurecidos ou escurecidos pela idade ${ }^{8}$.

Inúmeras técnicas podem ser escolhidas para a eliminação de manchas, por meio do uso de diferentes materiais. Dentre estes, podem ser utilizados as pastas formadas pela associação de ácido hidroclorídrico e pedra-pomes, ácido fosfórico 37\% e pedra-pomes ou, ainda, pela pasta que contém ácido clorídrico e micropartículas de carbeto de silício solúvel em água ${ }^{9-11}$.

Portanto, relevando os diferentes produtos existentes no mercado, este trabalho tem por objetivo relatar um procedimento clínico realizado através da microabrasão do esmalte no elemento 23 com a presença de mancha branca idiopática.

\section{Relato de Caso}

Paciente A. Z., 22 anos, sexo feminino, leocoderma, compareceu a Clínica de Dentística II da Universidade Federal da Paraíba com queixa do aspecto estético do elemento 23, devido a presença de mancha branca. A mesma relatou não lembrar quando a mancha branca apareceu e não saber a causa.

$\mathrm{Na}$ anamnese observou-se nada digno de nota. No exame clínico intra-oral, verificou-se a presença de manchas brancas na face vestibular e com um pequeno envolvimento da face interpoximal (mesial) do elemento 23 (Figura 1), com alteração de grau variável na translucidez do esmalte. O esmalte defeituoso apresentou espessura normal e superfície lisa e, foi possível identificar o limite entre o esmalte defeituoso e o esmalte normal.

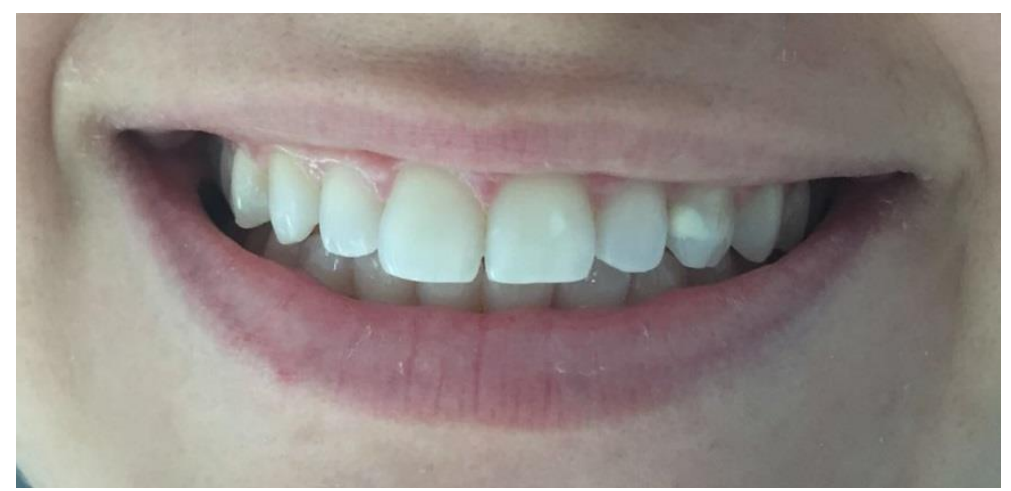

Figura 1: Aspecto inicial da mancha branca no elemento 23.

Para avaliar a profundidade da mancha foi utilizado o método de transiluminação dental, ou seja, utilização do fotopolimerizador posicionado na face palatina do referido elemento dentário 
(Figura 2). Com esse método, pode-se perceber que as manchas eram pouco profundas. Sendo assim, o diagnóstico sugestivo desse caso foi de opacidade demarcada idiopática.

Optou-se pelo tratamento através da Microabrasão do Esmalte Dental com o uso de Ácido clorídrico a $12 \%$ + Carbeto de silício, por ser um composto indicado para remoção química e mecânica controlada do esmalte manchado por hipoplasias, opacidades, fluorose, manchas brancas de cárie inativa, defeitos estruturais que pigmentam o esmalte e, por apresentar evidências cientificas de resultados estéticos satisfatórios. É ainda considerada uma técnica segura, conservadora, de fácil execução e baixo custo.

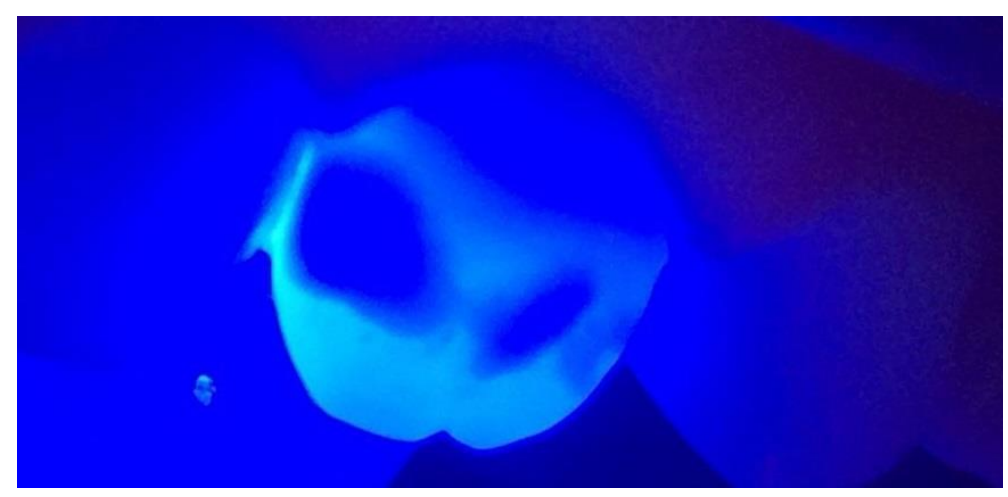

Figura 2: Método de transiluminação para avaliação da profundidade da mancha.

Após a avaliação da profundidade das manchas, foi feita a profilaxia do elemento com a pasta de pedra pomes e água, usando taça de borracha em baixa rotação. Em seguida, foi feito o isolamento absoluto, recobrindo as narinas da paciente, aplicado o topdam para servir de barreira de uma possível penetração da pasta microabrasiva pela interface lençol, dente e gengiva e colocado óculos de proteção no paciente.

Inicialmente foi posicionada a pasta microabrasiva na mancha presente na face vestibular com a ponta aplicadora do produto, seguido do início do processo de microabrasão com auxílio de espátula plástica, brunindo mecanicamente por 15 segundos sobre a mancha (Figura 3). Após, removeu-se o composto microabrasivo e a superfície foi lavada com água por 20 segundos, deixando um aspecto úmido, a fim de verificar a aparência da mancha após o microabrasionamento. Foi dado continuidade as aplicações, da mesma forma descrita acima, totalizando 5 aplicações de 15 segundos. A partir 6a aplicação, optou-se pela utilização do abrasionamento rotatório com auxílio de ponta de silicone acoplada ao micromotor (Figura 4), em 
virtude da mancha apresentar-se com aspecto puntiforme, seguindo a mesma técnica e totalizando 15 aplicações de 15 segundos na mancha da face vestibular.

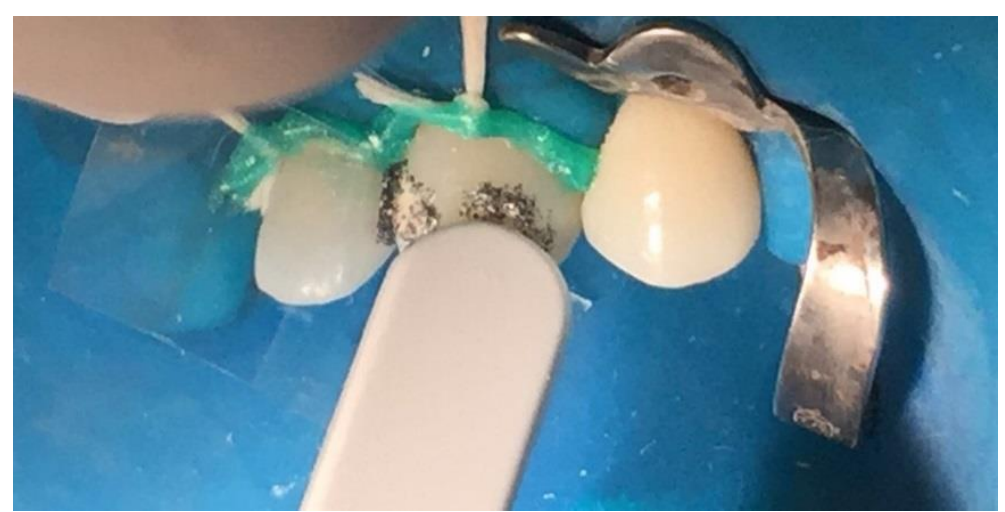

Figura 3: $1^{\mathrm{a}}$ aplicação da pasta microabrasiva com espátula, após isolamento absoluto, estabilização com fio dental e barreira gengival.

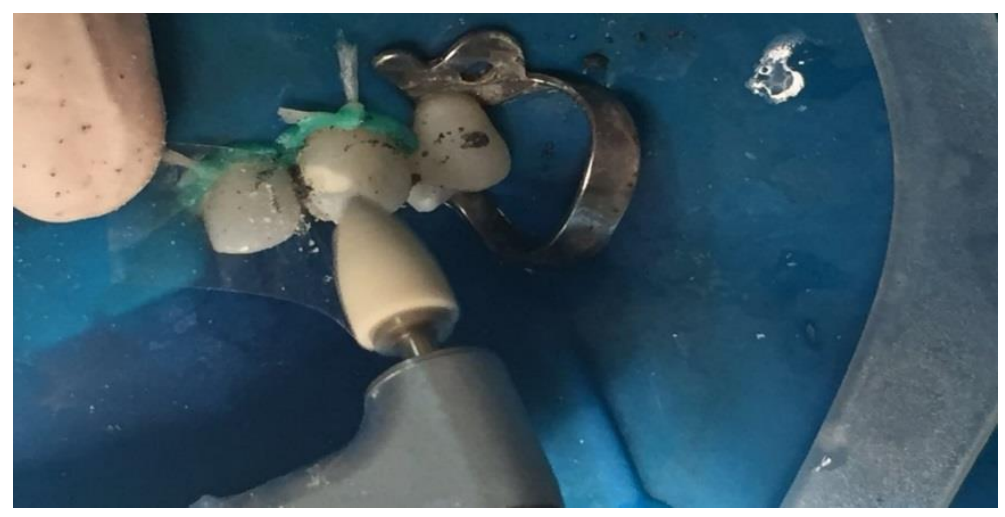

Figura 4: $6^{\mathrm{a}}$ aplicação com o uso da ponta de silicone acoplada ao micromotor de forma puntiforme.

Posteriormente, nesta mesma sessão clínica, foi dado início a aplicação da pasta microabrasiva na macha presente na face mesial. Para esta fase utilizou-se uma tira de polimento dental fina e flexível, associada a pasta microabrasiva, a fim de se obter um melhor contato do produto com a área do esmalte manchado. Foram feitas 5 aplicações de 15 segundos cada, com movimentos de serradura e fricção, sempre aplicando a mesma técnica de microabrasionar e lavar a superfície do esmalte entre as aplicações. Ao final, observou-se que a mancha não era visível clinicamente com o esmalte úmido e que a mesma tinha regredido, obtendo um bom resultado estético. 
Ao término da sessão clínica de microabrasionamento das duas manchas, foi feito o polimento do esmalte realizado com disco de feltro e pasta de polimento, seguido da aplicação tópica de flúor gel neutro realizada por 4 minutos. Em seguida procedeu-se a remoção do isolamento absoluto e, a paciente foi orientada a evitar ingerir alimentos que contêm corantes.

Decorridos 7 dias da primeira sessão, a paciente retornou a clínica e novo exame foi realizado. Observamos que a mancha havia reduzido, tendo alcançado aspecto clínico e estético satisfatório em relação as manchas apresentadas inicialmente. A paciente ficou satisfeita com o resultado do tratamento estético e conservador alcançado pela microabrasão, sendo finalizado e avaliado como sucesso clínico (Figura 5).

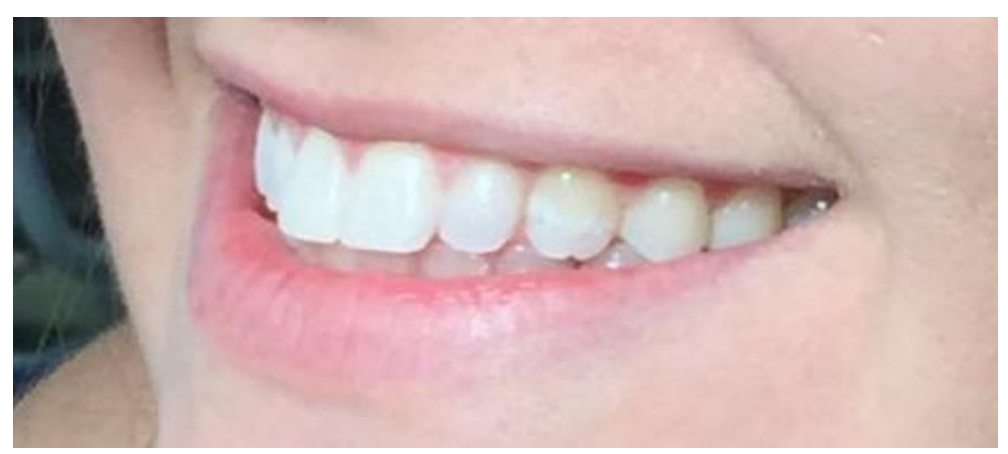

Figura 5: Aspecto clínico final.

\section{Discussão}

Atualmente, são vários os motivos que justificam o uso da microabrasão como uma técnica conservadora, segura, de fácil execução e resultado estético satisfatório. No entanto, no passado, a remoção de manchas no esmalte era tratadas através de preparos nos dentes e, posteriormente restauração. O uso da microabrasão tem sido bastante utilizada na Odontologia conservadora por ser uma técnica eficaz, quando se trata de remoção de manchas superficiais do esmalte ${ }^{12}$.

A escolha da técnica foi baseada nas características clínicas encontradas e o número de aplicações na técnica foi diretamente relacionado à profundidade e à quantidade de estrutura de esmalte envolvido pelas manchas o que vai de encontro com estudo ${ }^{13}$ em que escolheram a técnica de microabrasão do esmalte para o tratamento de manchas em um paciente com fluorose dental e, a quantidade de aplicações estava relacionada com a profundidades das manchas, na 
qual a técnica mostrou-se simples, rápida e sem inconvenientes ou risco para o paciente, simultaneamente eficaz na remoção das manchas.

Em relação ao produto e à técnica empregada na resolução do caso clínico, devemos seguir um protocolo clínico preestabelecido de proteção ao paciente, operador e auxiliar ${ }^{14}$. A consistência do produto selecionado Whiteness RM (FMG), composto de um gel (60\% do composto), que contém o ácido clorídrico a 12\%, e o abrasivo (40\% do composto), carbeto de silício, permitiu aos operadores maior controle sobre o material durante a sua aplicação e, foi escolhido devido as manchas serem um pouco profundas. A desvantagem é que esse produto desgasta mais estrutura dentária e é mais caústico quando comparado com outros materiais, como por exemplo o ácido fosfórico a $37 \%$ e pedra pomes ${ }^{1}$.

Conforme os procedimentos clínicos realizados, pode-se perceber que o protocolo inicial vai de acordo com estudo², na qual antes de começar a aplicação do material abrasivo é de fundamental importância o isolamento absoluto, proteção dos tecidos moles, uso do óculos de proteção tanto no paciente como no operador, em virtude do efeito caústico do ácido clorídrico durante a execução da técnica.

O material microabrasivo foi aplicado inicialmente nas manchas presente na face vestibular, restringindo-se ao local em que a mancha estava localizada e, isso corrobora com o estudo $^{5}$, no qual o material microabrasivo deve ser aplicado de forma pontual onde estão as manchas para evitar que o mesmo cause a erosão de tecido dental hígido.

A técnica de microabrasão foi realizada apenas em uma sessão clínica, pois conseguiu obter um bom resultado. Ao se realizar a técnica de microabrasão sem o clareamento dental, pode haver a necessidade de mais de uma sessão para a total eliminação das manchas e uniformização da cor, pois o mecanismo de ação da microabrasão do esmalte é extrínseco, sendo restrito às camadas mais superficiais do esmalte ${ }^{3}$. Mas com apenas uma sessão no presente caso clínico, foi possível obter uma resposta positiva. O clareamento dental para o caso foi descartado, pois evidenciaria ainda mais o aspecto branco da mancha.

Ao final da primeira sessão clínica foi aplicado flúor gel neutro no elemento dentário a fim de diminuir a sensibilidade pós-operatória. Em um caso clínico realizado, o flúor gel foi aplicado após a primeira sessão de microabrasão com a finalidade de reduzir os riscos de sensibilidade pós-operatória que pode ser decorrente da pressão exercida durante 0 ato operatório ou da localização da mancha ${ }^{8}$.

No caso clínico em questão devido as manchas serem pouco profundas, a técnica escolhida não removeu totalmente a lesão. A indicação da técnica de microabrasão deve levar em 
consideração à profundidade da lesão branca, que pode ter extensão mais superficial ou em maior profundidade no esmalte ${ }^{1}$. Tal diagnóstico implica diretamente no prognóstico do caso, visto que em casos de lesões brancas profundas, a microabrasão promove apenas discreta melhora no aspecto clínico, e não remove totalmente a lesão, o que deve ser explicado e discutido junto ao paciente.

Muitas técnicas vêm preconizadas para a remoção de manchas do esmalte dental, todas com o mesmo princípio de ação, ou seja: desmineralização e abrasão da superfície do esmalte manchado para exposição de uma camada subsuperficial sem alteração de cor, em uma única sessão. Dessa forma, nota-se que o prognóstico da remoção de manchas por microabrasão do esmalte é extremamente dependente de sua profundidade.

\section{Conclusão}

Considerando o resultado clínico obtido, podemos concluir que a técnica de microabrasão empregada atendeu a necessidade estética da paciente, e demonstrou ser eficaz, conservadora e segura para remoção de manchas superficiais no esmalte dental.

\section{Referências}

1. Oliveira LKM, Carvalho LAO, Assunção IV, Borges BCD, Santos AJS, Carvalho, WL, Dantas EDV. Microabrasão na estética dentária: sucesso com procedimento minimamente invasivo. Revista Ciência Plural. 2015;1(3):76-84.

2. Hoeppner MG, Araújo CSA, Carvalho MS. Microabrasão do esmalte dental: relato de um caso clínico. UFES Rev. Odontol. 2007; 9(2):51-56.

3. Castro ALS, Mendes CMC. Microabrasão e clareamento em dentes com fluorose: relato de um caso clínico. Rev. Ciênc. Méd. Biol. 2014; 13(3): 403-408.

4. Hintz R, Wagner S, Ruiz L. Microabrasão associada ao clareamento dental no tratamento da hipoplasia de esmalte. Caderno de Odontologia Clínica. 2017; 8(30):118-123.

5. Prado RNS, Zeidan LC, Lotufo MA, Rodrigues JA. Limites da microabrasão do esmalte dental relato de caso. Revista Saúde. 2014, 8(1/4): 30-36.

6. Gomes RP, Montenegro G. Microabrasão do esmalte associada ao clareamento dental. Revista Odontológica do Planalto Central. 2011; 2(1):16-21. 
7. Queiroz VAO, Martins GC, Zander-Grande C, Gomes JC, Campanha NH, Jorge JH. Report of two microabrasion techniques of enamel to remove stains and discussion. Rev Odontol UNESP. 2010; 39(6):369-372.

8. Peruchi CMS, Barreto Bezerra AC, Azevedo, TDPM, Barbosa e Silva E. O uso da microabrasão do esmalte para remoção de manchas brancas sugestivas de fluorose dentária:caso clínico. Revista Odontológica de Araçatuba. 2004; 25(2):72-77.

9. Allen K, Agosta C, Estafan D. Using microabrasive material to remove fluorose stains. J Am Dent Assoc. 2004; 135(3):319-23.

10. Ardu S, Stavridakis M, Krejci I. A minimally invasive treatment of severe dental fluorosis. Quintessence Int. 2007; 38(6):455-458.

11. Meireles SS, Andre DA, Leida FL, Bocangel JS, Demarco FF. Surface roughness and enamel loss with two microabrasion techniques. J Contemp Dent Pract. 2009;10(1):58-65.

12. Hermes SN. Microabrasão do esmalte dental para tratamento de fluorose. Rev Gaúcha Odontol. 2013; 61(0):427-433.

13. Freitas ESP, Diogo EF, Roselino PL. Microabrasão do esmalte como tratamento conservador da fluorose dental. Revista Científica da Universidade de Franca. 2006;6(1):41-46.

14. Conceição EN. Dentística, saúde e estética. 14th ed. Artes Médicas Sul; 2000, 124p. 